C 2021 IEEE. Personal use of this material is permitted. Permission from IEEE must be obtained for all other uses, in any current or future media, including reprinting/republishing this material for advertising or promotional purposes, creating new collective works, for resale or redistribution to servers or lists, or reuse of any copyrighted component of this work in other works.

Digital Object Identifier $\quad \underline{10.1109 / E C C E 47101.2021 .9595126 ~}$

2021 IEEE Energy Conversion Congress and Exposition (ECCE)

Analysis of Overmodulation in Power Synchronization-based Voltage Source Converters

Federico Cecati

Sante Pugliese

Marco Liserre

Xiongfei Wang

Frede Blaabjerg

\title{
Suggested Citation
}

F. Cecati, S. Pugliese, M. Liserre, X. Wang and F. Blaabjerg, "Analysis of Overmodulation in Power Synchronization-based Voltage Source Converters," 2021 IEEE Energy Conversion Congress and Exposition (ECCE), 2021. 


\section{Analysis of Overmodulation in Power Synchronization-based Voltage Source Converters}

\author{
Federico Cecati, Sante Pugliese and Marco Liserre \\ Chair of Power Electronics \\ Kiel University \\ Kiel, Germany \\ fc@tf-uni.kiel.de, sapu@tf-uni.kiel.de, ml@tf-uni.kiel.de
}

\author{
Xiongfei Wang and Frede Blaabjerg \\ Department of Energy Technology \\ Aalborg University \\ Aalborg, Denmark \\ xwa@et.aau.dk,fbl@et.aau.dk
}

\begin{abstract}
Low power system inertia in power electronics-dominated grids is a widely discussed problem. Power synchronization allows to emulate inertia by means of the active power low-pass filter, slowing down the grid frequency excursions. Nevertheless, inertia emulation during grid disturbances results in high energy exchange with the dc-link, which brings large dc voltage excursions. When the dc voltage dips below the ac line voltage peak, the modulation index can become too high and make the converter unable to produce the requested ac voltage. The consequent saturation of the voltage can cause current control wind-up, harmonic distortion and loss of controllability. Moreover, when the reference voltage is saturated, the converter does not behave anymore according to the power synchronization control law, making the conventional stability analyses not valid. Despite the relevance of these issues, a systematic analysis about the voltage saturation induced by grid disturbances in power converters is still missing. For that, this paper proposes a mathematical tool based on Bode plots. The target of this tool is to properly chose the converter parameters in order to tolerate grid disturbances without reaching the voltage saturation. Simulations and experiments are provided.
\end{abstract}

Index Terms-Power Synchronization, Virtual Inertia, Overmodulation, State-Space Modeling

\section{INTRODUCTION}

The evolution of the power generation from centralized towards distributed is pushing a change in the converter control strategy [1], [2]. Power synchronization control of Voltage Source Converters (VSCs) is gaining a prominent position in this change, for its ability to ensure converter stable operation also in absence of a main stiff grid [3], [4]. Power synchronization generally uses a low-pass filter in the measured active power both

The authors gratefully acknowledge the funding by Deutsche Forschungsgemeinschaft (DFG, German Research Foundation) via the Priority Program DFG SPP 1984 "Hybrid and Multimodal Energy Systems" and by Gesellschaft für Energie und Klimaschutz SchleswigHolstein $\mathrm{GmbH}$ (EKSH) doctoral studies grant. for harmonics filtering and for control loops stabilization [5], [6]. This filtering operation is responsible for the converter inertia emulation, proportionally to the filter time constant [5], [7]; inertia emulation results in energy exchange with the dc bus [8], which can bring a high dc voltage excursion.

When the dc voltage dips, the converter is not able to produce the desired reference voltage at the output terminals, if it becomes lower than the reference ac phase-to-phase inverter voltage. This phenomena is known as overmodulation and it causes the loss of converter controllability and high current distortion [9]. In order to avoid it, voltage limitation strategies and antiwindup current controllers have been proposed [9]-[11]. Nevertheless, when the voltage limitation is operational, the converter output ac voltage is different from the reference voltage produced by the control system. In that situation, the converter is not anymore operating as originally designed and there is no warranty that the stability is maintained for the entire duration of the grid disturbance. Thus, for a safe and stable power converter operation, it is desirable to avoid the dc voltage to dip below the ac phase-to-phase voltage, by properly designing the converter controller.

The occurrence of the voltage saturation depends on several factors, including dc nominal voltage, entity of the grid disturbance, dc voltage control bandwidth and frequency droop tuning among them. To have a deeper insight and understand how to address this problem, a mathematical tool for its study is necessary. Despite the relevance of this phenomena, such a tool has not been proposed in literature yet.

In this paper, a state-space model of a power synchronization-based converter including all the control loops and the dc-link dynamics is firstly derived and linearized around its operating point. From the mentioned model, the transfer functions from the grid 


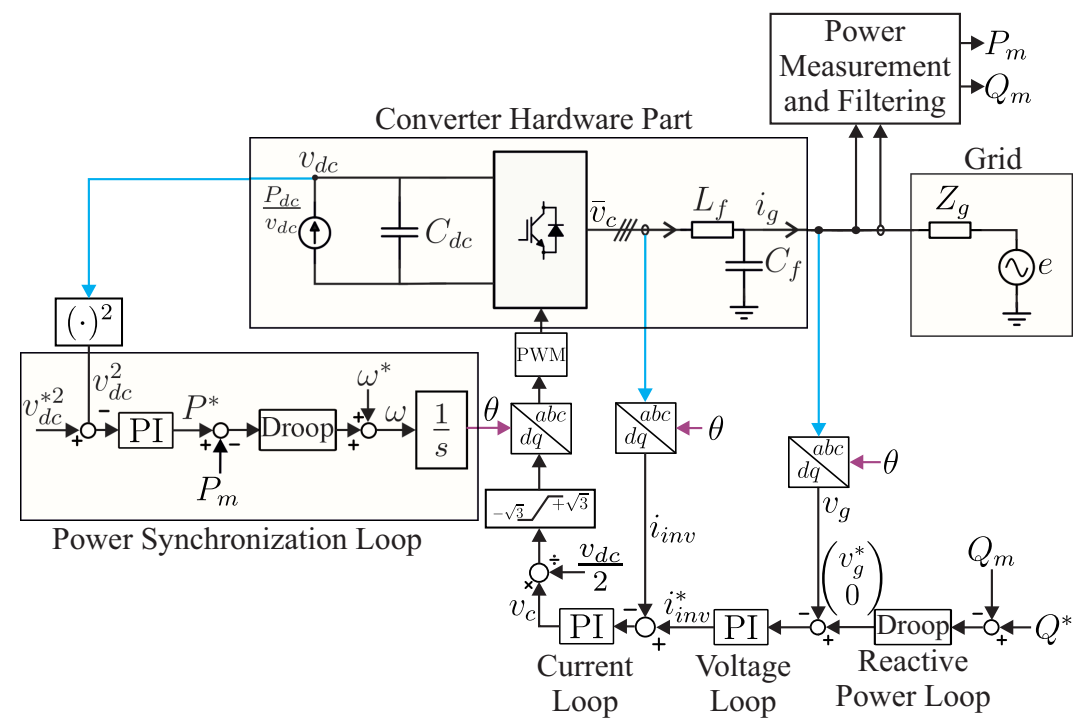

Figure 1: The considered three-phase power converter implementing power synchronization loop and reactive power loop.

disturbances to the modulation index are computed: the Bode plots of the latter transfer functions give a measure of the sensitivity of the modulation index with respect to the grid voltage and frequency disturbances at various frequencies. The higher the magnitude of the Bode plot, the higher the sensitivity and the higher the risk of reaching the voltage saturation after a grid disturbance. Simulations shows that the modulation index sensitivity evaluated through the transfer function is a good measure for the converter voltage saturation risk. The parameter variations, e.g. dc-link nominal voltage and frequency droop coefficients, allow to evaluate the influence of these parameters on the risk of voltage saturation, and to properly tune the controller in order to avoid it. Simulations and experimental results are carried out to validate the feasibility of the proposed analytic tool.

\section{System Description ANd OVERMOdUlation DISCUSSION}

The control scheme of the PWM power converter under analysis is depicted in Fig. 1. The synchronization is achieved through a power synchronization loop, composed of an outer dc-link voltage PI controller and an inner frequency droop controller [4]. The dc-link voltage control uses the square of the dc voltage $v_{d c}$ to make the control dynamics independent on the dc nominal voltage [12]. A droop control is used for the reactive power regulation, cascaded with a double voltage-current loop, similar to [6]. The measured active power $P_{m}$ and reactive power $Q_{m}$ used in the droop controls are the result of a low-pass filtering [6]. The filtering serves not only to eliminate the measurement noise, but its cutoff frequency has a correlation with the virtual inertia provided by the power converter [5].
The digital delay and the semiconductor switching dynamics are neglected in this study, since they have a much faster dynamics in respect to the dynamics of interest (the dc capacitor discharge). Under this assumption, according to Fig. 1, it yields

$$
v_{c}=\bar{v}_{c}
$$

during normal operating conditions, i.e. when the modulation index defined as the ratio between the peak-topeak line ac voltage and the dc voltage

$$
m_{i}=2 \sqrt{3} \frac{\sqrt{v_{c}^{T} v_{c}}}{v_{d c}}
$$

is lower than 1 . The expression $\sqrt{v_{c}^{T} v_{c}}$ is the amplitude of the $d q$ reference voltage $v_{c}$. If the modulation index $m_{i}$ becomes higher than 1 , the converter operates in the overmodulation region [10]. By using the third harmonic injection or different modulation techniques, the converter can keep the controllability and a good power quality even with $m_{i}>1$ for a certain range [13]. However, there exists in each case a modulation index critical value $\bar{m}_{i}$ above which the converter is not able to properly produce the reference voltage $v_{c}$ in the output terminals [10], [14]. In that case, the voltage $v_{c}$ is saturated, and the condition (1) does not yield anymore [10]. With saturated voltage, the converter does not behave anymore according to the designed control law, and its stability is difficult to predict. Furthermore, the voltage saturation introduces harmonic distortion, loss of converter controllability and even the problem of the integrators wind-up, which needs anti-windup current control strategies to be mitigated [9], [11].

For these reasons, the voltage saturation represents a 
risk for the converter stability and has to be avoided. The target of this paper is to investigate in which conditions and under which grid disturbances $v_{c}$ reaches the voltage saturation level and how to design and tune the converter in order to avoid it. The modulation index critical value is considered $\bar{m}_{i}=1$ in the following analysis, without loss of generality for the proposed method.

\section{Converter State-Space Modeling}

The converter and its control loops are modeled in the state-space through a set of nonlinear differential and algebraic equations [15]-[17] in the form:

$$
\left\{\begin{array}{l}
\dot{x}=f(x)+g(x)\left(\begin{array}{c}
E_{g} \\
f_{g}
\end{array}\right) \\
m_{i}=h(x)
\end{array}\right.
$$

where the grid voltage amplitude $E_{g}$ and frequency $f_{g}$ are set as disturbance input variables, expressed in per unit. The state vector in (3) is defined as $x=$ $\left(\begin{array}{llllllllll}i_{i} & v_{g} & \Phi_{1} & \Phi_{g} & v_{d c} & \Phi_{d c} & P_{m} & Q_{m} & \delta_{g} & i_{g}\end{array}\right)$. The variables $\Phi_{i n v}, \Phi_{g}$ and $\Phi_{d c}$ are the integral states of ac current, ac voltage and dc voltage PI controllers, respectively. The complete converter state-space model is composed of a set of differential equations

$$
\left\{\begin{array}{l}
\dot{i}_{1}=-\Omega i_{1}+\frac{1}{L_{f}} v_{c}-\frac{1}{L_{f}} v_{g} \\
\dot{v}_{g}=-\Omega v_{g}+C_{f} i_{1}-C_{f} i_{g} \\
\dot{\Phi}_{1}=i_{1}^{*}-i_{1} \\
\dot{\Phi}_{g}=v_{g}^{*}-v_{g} \\
\dot{v}_{d c}^{2}=-\frac{3}{2} \frac{1}{C_{d c}} v_{g}^{T} i_{g}+\frac{1}{C_{d c}} P_{d c} \\
\dot{\Phi}_{d c}=v_{d c}^{2}-v_{d c}^{* 2} \\
\dot{P}_{m}=-\omega_{f} P_{m}+\omega_{f} v_{g}^{T} i_{g} \\
\dot{Q}_{m}=-\omega_{f} Q_{m}+\omega_{f} v_{g}^{T}\left(\begin{array}{ll}
0 & -1 \\
1 & 0
\end{array}\right) i_{g} \\
\dot{\delta}^{2}=-m_{p}\left(P_{m}-P^{*}\right) \\
\dot{i}_{g}=-\frac{R_{g}}{L_{g}} i_{g}-\Omega i_{g}+\frac{1}{L_{g}}\left(v_{g}-T(\delta) T\left(\delta_{g}\right) E_{n}\left(\begin{array}{c}
E_{g} \\
0
\end{array}\right)\right) \\
\dot{\delta}_{g}=2 \pi f_{n}\left(f_{g}-1\right)
\end{array}\right.
$$

Table I: Simulation parameters for the power synchronization-based converter

\begin{tabular}{lc}
\hline Parameters & Values \\
\hline \hline Line-to-line grid voltage $e(\mathrm{~V})$ & 690 \\
Short Circuit Ratio of $Z_{g}$ & 1.5 \\
$R / X$ ratio of $Z_{g}$ & 0.3 \\
Nominal active power $P_{d c}(\mathrm{MW})$ & 1 \\
Nominal dc-link voltage $v_{d c}^{*}(\mathrm{~V})$ & 1200 \\
Switching frequency $(\mathrm{kHz})$ & 2 \\
DC-link capacitor $C_{d c}(\mathrm{mF})$ & 22 \\
Filter inductor $L_{f}(\mathrm{mH})$ & 0.1 \\
\hline
\end{tabular}

and a set of algebraic equations

$$
\left\{\begin{array}{l}
v_{c}=K_{p}\left(i_{1}^{*}-i_{1}\right)+K_{i} \Phi_{1} \\
i_{g}^{*}=K_{p_{v}}\left(v_{g}^{*}-v_{g}\right)+K_{i_{v}} \Phi_{g} \\
v_{g}^{*}=\left(\begin{array}{c}
E_{n}-n_{q}\left(Q_{m}-Q^{*}\right) \\
0
\end{array}\right) \\
P^{*}=K_{p_{d c}}\left(v_{d c}^{2}-v_{d c}^{* 2}\right)+K_{i_{d c}} \Phi_{d c} \\
T(\delta)=\left(\begin{array}{cc}
\cos \delta & \sin \delta \\
-\sin \delta & \cos \delta
\end{array}\right) \\
\Omega=\left(\begin{array}{cc}
0 & -\omega \\
\omega & 0
\end{array}\right)
\end{array}\right.
$$

$\omega_{f}$ is the power filter time constant, $E_{n}$ and $f_{n}$ are the grid voltage nominal amplitude and frequency respectively, $m_{p}$ is the $P-\omega$ droop gain, $n_{q}$ is the $Q-V$ droop gain and $v_{g}$ is the voltage across the capacitor $C_{f}$. The parameters $K_{p}$ and $K_{i}$ are the current controller proportional and integral gains, $K_{p_{v}}$ and $K_{i_{v}}$ the ac voltage controller proportional and integral gains, $K_{p_{d c}}$ and $K_{i_{d c}}$ are the dc voltage controller proportional and integral gains. $\Phi_{1}, \Phi_{v}$ and $\Phi_{d c}$ are the integral states of inner current loop, ac voltage controller and dc voltage controller, respectively. The modulation index $m_{i}$ is chosen as the output of the model (3), and it can be expressed through (2).

\section{A Mathematical Tool for the Study of THE Voltage SATURATION}

The first step to investigate the voltage saturation phenomena is to quantitatively study how the modulation index varies in response to grid disturbances. The saturation is more likely to occur when the modulation index is highly sensitive to grid disturbances, e.g. the converter reacts to a relatively small grid disturbance with a relatively large oscillation in the modulation index. The information about this sensitivity is enclosed in the input-output characteristic of the model (3), which indeed has been defined with the grid voltage and frequency as input and the modulation index as output. The modulation index expression (2) as well as 
Table II: Analytic results of the Bode analysis done in Fig. 2

\begin{tabular}{l|ccccc}
\hline \hline Frequency droop gain $m_{p}$ & $25 \cdot 10^{-6}$ & $20 \cdot 10^{-6}$ & $15 \cdot 10^{-6}$ & $10 \cdot 10^{-6}$ & $8 \cdot 10^{-6}$ \\
\hline$\widehat{W}_{E}$ & 1.87 & 1.62 & 1.46 & 1.46 & 1.58 \\
$\widehat{W}_{f}$ & 24.54 & 31.14 & 42.62 & 67.71 & 88.80 \\
\hline$\Delta m_{i}=\widehat{W}_{f} \Delta f_{g}$ & 0.15 & 0.19 & 0.25 & 0.4 & 0.53 \\
$m_{i, e q}+\Delta m_{i}$ & 0.73 & 0.77 & 0.83 & 0.98 & $\mathbf{1 . 1 1}$
\end{tabular}

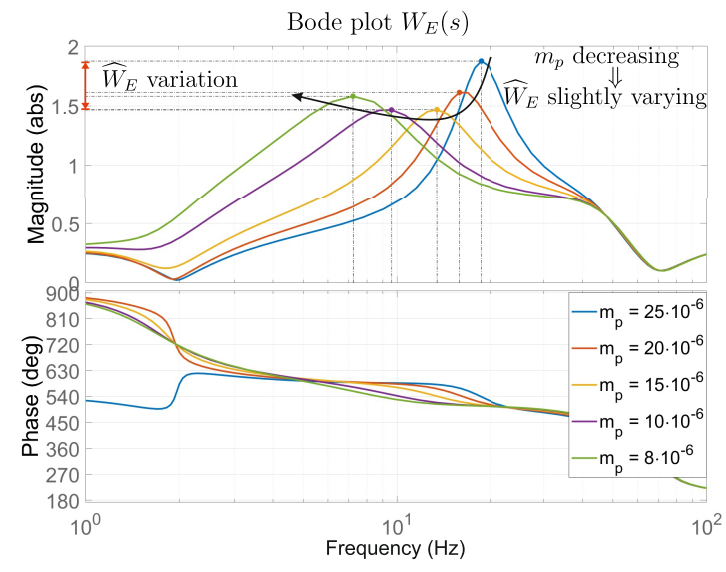

(a)

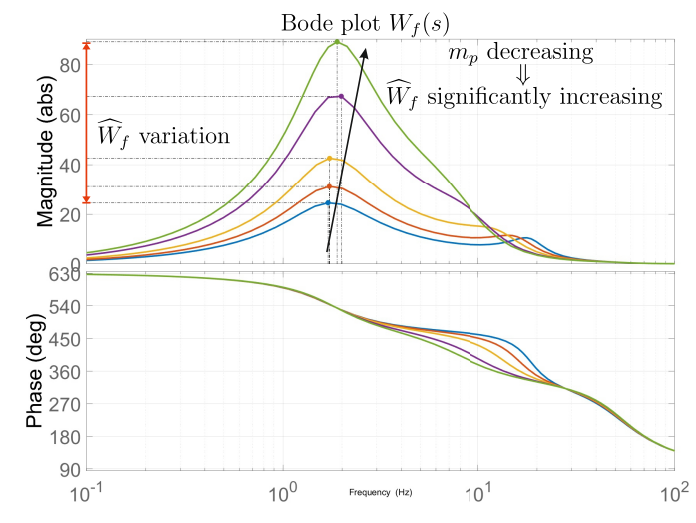

(b)

Figure 2: The proposed Bode analysis under different values of the frequency droop gain. (a) The frequency response from a voltage disturbance to the modulation index. (b) The frequency response from a frequency disturbance to the modulation index.

the saturation phenomena itself are nonlinear. However, the modulation index sensitivity in respect to smallsignal grid disturbances can be investigated also with linear analysis tools. It will be shown through simulation results that the modulation index sensitivity obtained through the proposed linear analysis is a good measure of the risk of voltage saturation in response to grid disturbances.

For the proposed study, the steady-state values of the state $x$ and modulation index $m_{i}$ are needed. The equi-
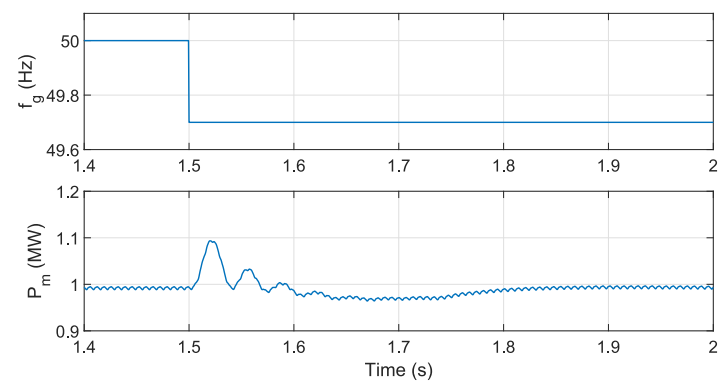

(a)

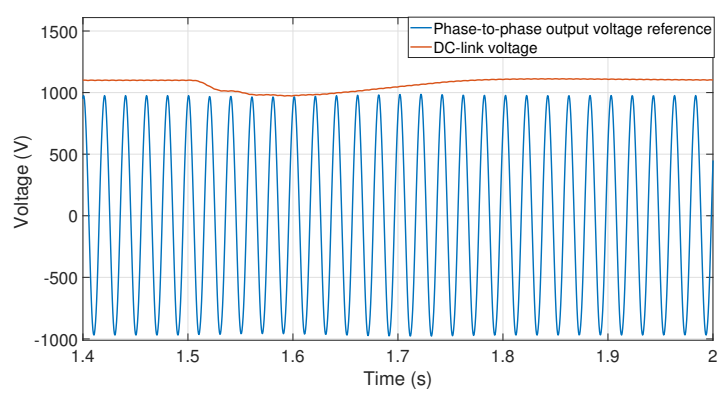

(b)

Figure 3: Simulation of a frequency dip for the power converter in the case of $m_{p}=10 \cdot 10^{-6}$. (a) The frequency dip and the converter active power (b) The converter dc and ac terminal voltages.

librium state $x_{e q}$ is computed by numerical integration of the nonlinear model in (3) in the grid nominal point defined by $E_{g}=1$ p.u., $f_{g}=1$ p.u. for a time $T$ large enough:

$$
x_{e q}=\int_{0}^{T} f(x)+g(x)\left(\begin{array}{l}
1 \\
1
\end{array}\right) d t
$$

The output function $h(x)$ is then evaluated in the equilibrium state $x_{e}$ computed through (6), obtaining the steady-state value of the modulation index

$$
m_{i, e q}=h\left(x_{e q}\right)
$$

The nonlinear model in (3) is linearised through Jacobian matrices computation, obtaining:

$$
\left\{\begin{array}{l}
\dot{x}=A x+B\left(\begin{array}{c}
E_{g} \\
f_{g}
\end{array}\right) \\
m_{i}=C x
\end{array}\right.
$$



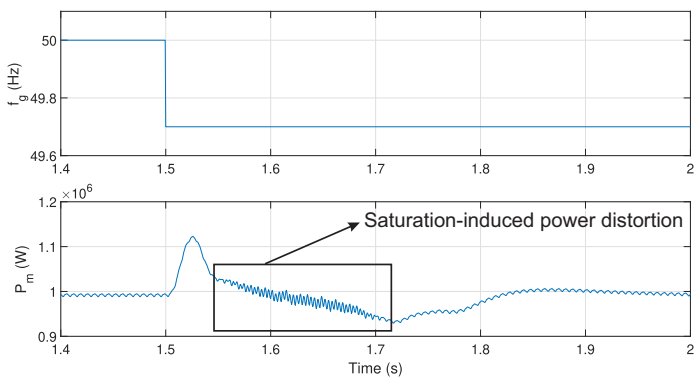

(a)

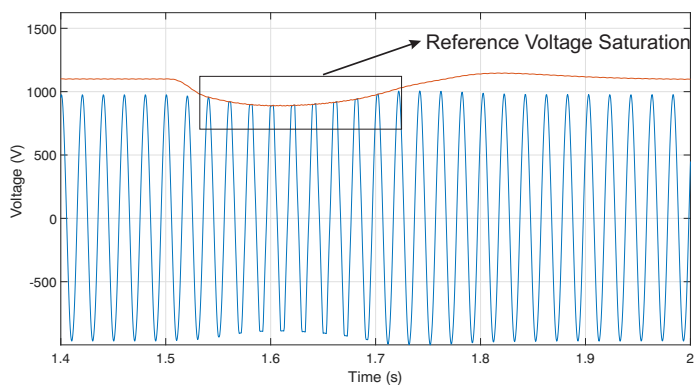

(b)

Figure 4: Simulation of a frequency dip for the power converter in the case of $m_{p}=8 \cdot 10^{-6}$. (a) The frequency dip and the converter active power (b) The converter dc and ac terminal voltages.

From the linearised model (8), the transfer function $W(s)$ can be computed as $W(s)=C(s I-A)^{-1} B$.

$$
m_{i}=W(s)\left(\begin{array}{c}
E_{g} \\
f_{g}
\end{array}\right)=\left(\begin{array}{ll}
W_{E}(s) & W_{f}(s)
\end{array}\right)\left(\begin{array}{c}
E_{g} \\
f_{g}
\end{array}\right)
$$

The transfer function $W(s)$ is a $1 \times 2$ matrix: the element $W_{E}(s)$ describes how a disturbance in the voltage amplitude $E_{g}$ reflects on the modulation index $m_{i}$, while $W_{f}(s)$ describes the influence of a grid frequency $f_{g}$ disturbance on $m_{i}$. The peak magnitudes of $W_{E}(s)$ and $W_{f}(s)$, highlighted with the colored dot in Fig. 2, are denominated $\widehat{W}_{E}$ and $\widehat{W}_{f}$ respectively, and given in Table II. They represent the maximum gain from a generic grid disturbance to the modulation index, and give therefore a measure for the modulation index sensitivity: the higher the peak magnitudes, the more sensitive the modulation index to grid disturbances, the higher the risk of voltage saturation. Being this analysis based on the linearised system, the obtained results are accurate for small grid disturbances $\left( \pm 0.15\right.$ p.u. for $E_{g}$, $\pm 0.5 \mathrm{~Hz}$ for $f_{g}$ ) and loose progressively the accuracy for larger disturbances.

A 1 MW power converter as in Fig. 1 is modeled according to (3), linearised, and the its transfer function $W(s)$ as defined in (9) is computed. The converter and grid parameters are summarized in Table I.

In Fig. 2, $W(s)$ is plotted for different values of $m_{p}$, to see its influence on the modulation index sensitivity. Table II reports the magnitude peak values of $W_{E}(s)$ and $W_{f}(s)$ in Fig. 2. By decreasing $m_{p}$, the equivalent inertia provided by the converter increases [5] and higher active power is injected during the frequency dips. Therefore, the dc capacitor discharges more, increasing the risk of voltage saturation. This intuition is confirmed by the Bode plot of $W_{f}(s)$ in Fig. 2 where the peak magnitude increases when $m_{p}$ decreases. Conversely, $W_{E}(s)$ does not vary significantly with $m_{p}$.

Table III: Back-to-back converter and power amplifier parameters

\begin{tabular}{lc}
\hline Parameters & Values \\
\hline \hline Line-to-line power amplifier voltage $e(\mathrm{~V})$ & 430 \\
Power amplifier inductance $Z_{g}(\mathrm{mH})$ & 7.5 \\
$R / X$ ratio of $Z_{g}$ & 0.3 \\
Power rating $P_{d c}(\mathrm{~kW})$ & 4 \\
DC-link voltage $v_{d c}^{*}(\mathrm{~V})$ & 700 \\
Switching frequency $(\mathrm{kHz})$ & 10 \\
DC-link capacitor $C_{d c}(\mathrm{mF})$ & 1.2 \\
Filter inductor $L_{f}(\mathrm{mH})$ & 5.5 \\
\hline
\end{tabular}

\section{Simulation Results}

The Bode analyses in Fig. 2 and Table II reveal if the converter is able to support a given grid disturbance without reaching voltage saturation with different values of $m_{p}$. Considering a frequency dip $\Delta f_{g}=0.006$ p.u., corresponding to $0.3 \mathrm{~Hz}$, the consequent modulation index deviation $\Delta m_{i}$ is computed as $\widehat{W}_{f} \Delta f_{g}$ and reported in Table II for different values of $m_{p}$. The steady state value of the modulation index, computed through (6), is $m_{i, e q}=0.58$. From $m_{i, e q}$, if the modulation index deviation $\Delta m_{i}$ is high enough to bring $m_{i}$ above 1, the converter will reach the voltage saturation during the disturbance. Table II report the value of $m_{i, e q}+\Delta m_{i}$ which becomes higher than 1 only in the case $m_{p}=8 \cdot 10^{-6}$, marked in bold font. The simulations in Figs. 3 and 4 are realized to validate the analytic results of Table II. A frequency dip $\Delta f_{g}=0.006$ is simulated at the instant $t=1.5 \mathrm{~s}$ and the converter response is evaluated in the cases $m_{p}=10 \cdot 10^{-6}$ and $m_{p}=8 \cdot 10^{-6}$, according to the mathematical analysis reported in Table II. In Fig. 3, the dc capacitor $C_{d c}$ discharges during the frequency dip, but not enough to go below the ac terminal voltage and trigger the voltage saturation. Different is the case of Fig. 4 , where the dip results in a modulation index deviation $\Delta m_{i}$ large enough to trigger the saturation. Indeed, ac voltage reference saturation due to overmodulation can be observed in Fig. 4(b). The consequence of the voltage saturation is the output power distortion, evident in Fig. 4(a). The phenomena of windup is avoided thanks to a anti-windup strategy implemented in the current control [9]. The simulation results in Figs. 3 and 4 are in 


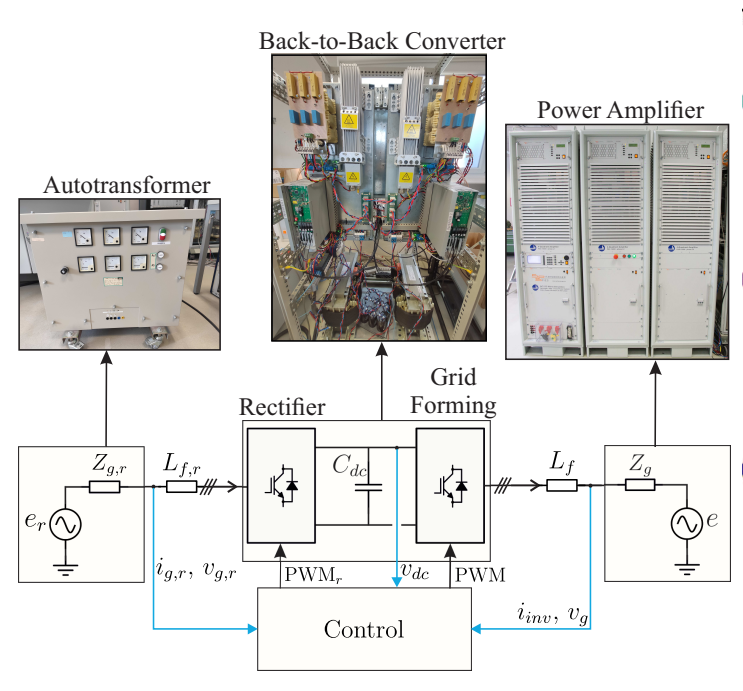

(a)

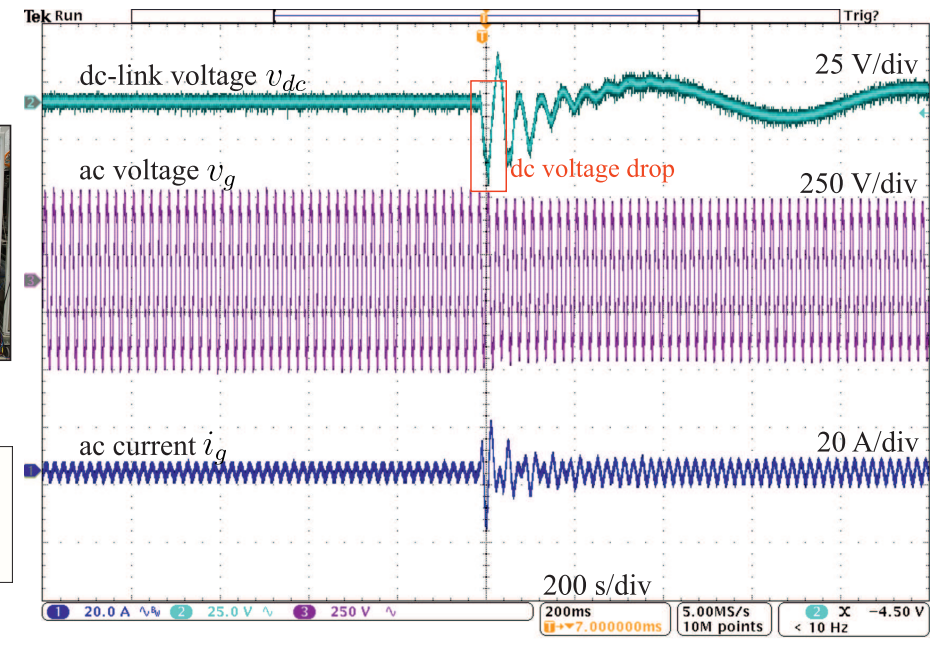

(b)

Figure 5: Experimental results. (a) The experimental setup. (b) Grid voltage sag in the considered experimental setup.

accordance with the analytic results in Fig. 2 and Table II.

It is important to notice that with the tuning $m_{p}=$ $8 \cdot 10^{-6}$, whereby the converter reaches the voltage saturation level, the converter is small-signal stable. That reveals that voltage saturation and small-signal stability are two distinct aspects: a controller tuning, which implies good and robust small-signal stability has no implication on the risk of voltage saturation, and vice versa. Therefore, it is important to consider the voltage saturation risk as a target in the converter tuning and design, in particular when implementing virtual inertia and active power support strategies, which cause wide fluctuations of the dc voltage and it is potentially dangerous for the voltage saturation [18], [19].

\section{EXPERIMENTAL RESULTS}

The converter in Fig. 1 is reproduced in the lab with a $4 \mathrm{~kW}$ scaled-down setup, as shown in Fig. 5(a). The dc power $P_{d c}$, modeled in Fig. 1 as a current source, is generated by a rectifier connected in back-toback configuration with the considered VSC. A DSpace ${ }^{\circledR}$ MicroLabBox is used to control the back-to-back converter. A voltage sag of 0.2 p.u. is applied in the power amplifier, and dc voltage, ac voltage and ac current are shown in Fig. 5(b). In the instant of the sag, the dc voltage rapidly decrease, before being restored from the control to its nominal value. In case the dc voltage drop is large enough, voltage saturation can occur and jeopardize the converter integrity and stability.

\section{CONCLUSION}

In this paper, the voltage saturation phenomena triggered by grid disturbances are studied starting from the complete state-space model of a power synchronizationbased power converter. The transfer functions from the grid voltage magnitude to the modulation index, $W_{E}(s)$, and from the grid frequency to the modulation index, $W_{f}(s)$, are derived. The higher the peak magnitudes of these two transfer functions, the more sensitive the modulation index to the grid voltage and frequency variations, and the higher the risk of voltage saturation. Different values of the frequency droop coefficient result in different modulation index sensitivity, providing a guideline to tune this parameter with the consideration of voltage saturation. Simulation results show furthermore that voltage saturation and small-signal instability are two different concepts: a converter can suffer from the voltage saturation problem due to not proper controller tuning even when the used parameters ensures a good and robust small-signal stability.

\section{REFERENCES}

[1] F. Blaabjerg, Y. Yang, D. Yang, and X. Wang, "Distributed powergeneration systems and protection," Proceedings of the IEEE, vol. 105, no. 7, pp. 1311-1331, 2017.

[2] R. Teodorescu, M. Liserre, and P. Rodriguez, Grid converters for photovoltaic and wind power systems. John Wiley \& Sons, 2011, vol. 29.

[3] J. Rocabert, A. Luna, F. Blaabjerg, and P. Rodriguez, "Control of power converters in ac microgrids," IEEE transactions on power electronics, vol. 27, no. 11, pp. 4734-4749, 2012.

[4] L. Zhang, L. Harnefors, and H. Nee, "Power-synchronization control of grid-connected voltage-source converters," IEEE Transactions on Power Systems, vol. 25, no. 2, pp. 809-820, 2010.

[5] S. D'Arco and J. A. Suul, "Equivalence of virtual synchronous machines and frequency-droops for converter-based microgrids," IEEE Transactions on Smart Grid, vol. 5, no. 1, pp. 394-395, 2014.

[6] N. Pogaku, M. Prodanovic, and T. C. Green, "Modeling, anal- 
ysis and testing of autonomous operation of an inverter-based microgrid," IEEE TPEL, vol. 22, no. 2, pp. 613-625, 2007.

[7] L. Harnefors, M. Hinkkanen, U. Riaz, F. M. M. Rahman, and L. Zhang, "Robust analytic design of power-synchronization control," IEEE Transactions on Industrial Electronics, vol. 66, no. 8, pp. 5810-5819, 2019.

[8] Q.-C. Zhong and G. Weiss, "Synchronverters: Inverters that mimic synchronous generators," IEEE Transactions on industrial electronics, vol. 58, no. 4, pp. 1259-1267, 2010.

[9] A. G. Yepes, J. Doval-Gandoy, and H. A. Toliyat, "Multifrequency current control including distortion-free saturation and antiwindup with enhanced dynamics," IEEE Transactions on Power Electronics, vol. 33, no. 9, pp. 7309-7313, 2018.

[10] R. Ottersten and J. Svensson, "Vector current controlled voltage source converter-deadbeat control and saturation strategies," IEEE Transactions on Power Electronics, vol. 17, no. 2, pp. 279285, 2002.

[11] C. Bohn and D. Atherton, "An analysis package comparing pid anti-windup strategies," IEEE Control Systems Magazine, vol. 15, no. 2, pp. 34-40, 1995

[12] L. Zhang, L. Harnefors, and H. Nee, "Interconnection of two very weak ac systems by vsc-hvdc links using power-synchronization control," IEEE Transactions on Power Systems, vol. 26, no. 1, pp. 344-355, 2011.

[13] R. Lyra and T. Lipo, "Torque density improvement in a six- phase induction motor with third harmonic current injection," IEEE Transactions on Industry Applications, vol. 38, no. 5, pp. 1351-1360, 2002.

[14] B. Wu and M. Narimani, High-power converters and AC drives. John Wiley \& Sons, 2017.

[15] F. Cecati, R. Zhu, M. Langwasser, M. Liserre, and X. Wang, "Scalable state-space model of voltage source converter for lowfrequency stability analysis," in 2020 IEEE Energy Conversion Congress and Exposition (ECCE), 2020, pp. 6144-6149.

[16] F. Cecati, R. Zhu, M. Liserre, and X. Wang, "State-feedbackbased low-frequency active damping for vsc operating in weakgrid conditions," in 2020 IEEE Energy Conversion Congress and Exposition (ECCE), 2020, pp. 4762-4767.

[17] F. Cecati, M. Andresen, R. Zhu, Z. Zou, and M. Liserre, "Robustness analysis of voltage control strategies of smart transformer," in IECON 2018 - 44th Annual Conference of the IEEE Industrial Electronics Society, 2018, pp. 5566-5573.

[18] S. Yang, J. Fang, Y. Tang, H. Qiu, C. Dong, and P. Wang, "Modular multilevel converter synthetic inertia-based frequency support for medium-voltage microgrids," IEEE Transactions on Industrial Electronics, vol. 66, no. 11, pp. 8992-9002, 2019.

[19] J. Fang, H. Li, Y. Tang, and F. Blaabjerg, "Distributed power system virtual inertia implemented by grid-connected power converters," IEEE Transactions on Power Electronics, vol. 33, no. 10 , pp. 8488-8499, 2017. 